\title{
Clinical characteristics and predictors of death among hospitalized patients infected with SARS-CoV-2 in Sicily, Italy: A retrospective observational study
}

\author{
FEDERICA COSENTINO $^{1}$, VITTORIA MOSCATT ${ }^{1}$, ANDREA MARINO $^{1}$, ALESSIO PAMPALONI $^{1}$, \\ DANIELE SCUDERI ${ }^{1}$, MANUELA CECCARELLI ${ }^{1}$, FRANCESCO BENANTI ${ }^{1}$, MARIA GUSSIO ${ }^{1}$, \\ LICIA LAROCCA $^{1}$, VINCENZO BOSCIA ${ }^{1}$, GIOVANNI VINCI ${ }^{1}$, ALDO ZAGAMI ${ }^{1}$, ANNA ONORANTE ${ }^{1}$, \\ GAETANO LUPO ${ }^{1}$, SALVATORE TORRISI $^{1}$, SILVANA GRASSO ${ }^{1}$, ROBERTO BRUNO ${ }^{1}$, CARMELO IACOBELLO $^{2}$, \\ SALVATORE BONFANTE ${ }^{3}$, LUIGI GUARNERI ${ }^{4}$, ANTONIO CASCIO ${ }^{5}$, ANTONELLA FRANCO ${ }^{6}$, \\ ROSSELLA FONTANA DEL VECCHIO ${ }^{6}$, MARIA ANTONIETTA DI ROSOLINI ${ }^{7}$, ALFREDO PULVIRENTI ${ }^{8}$, \\ DAMIANO LARNE $^{9}$, GIUSEPPE NUNNARI ${ }^{9}$, BENEDETTO MAURIZIO CELESIA ${ }^{1}$ and BRUNO CACOPARDO ${ }^{1}$ \\ ${ }^{1}$ Unit of Infectious Diseases, Department of Clinical and Experimental Medicine, ARNAS Garibaldi Nesima Hospital, \\ University of Catania, I-95122 Catania; ${ }^{2}$ Infectious Disease Unit, Cannizzaro Hospital, I-95126 Catania; \\ ${ }^{3}$ Infectious Diseases Unit, Gravina Hospital, I-95041 Caltagirone, Catania; ${ }^{4}$ Infectious Diseases Unit, Enna Hospital, \\ I-94100 Enna; ${ }^{5}$ Infectious and Tropical Diseases Unit, Department of Health Promotion Sciences Maternal and \\ Infantile Care, Internal Medicine and Medical Specialties (PROMISE), University of Palermo, I-90127 Palermo; \\ ${ }^{6}$ Infectious Diseases Unit, Siracusa Hospital, I-96100 Siracusa; ${ }^{7}$ Infectious and Tropical Diseases Unit, \\ Modica Hospital, I-97015 Ragusa; ${ }^{8}$ Bioinformatics Section, Department of Clinical and Experimental Medicine, \\ University of Catania, I-95125 Catania; ${ }^{9}$ Unit of Infectious Diseases, Department of Clinical and \\ Experimental Medicine, University of Messina, I-98124 Messina, Italy
}

Received December 3, 2021; Accepted February 2, 2022

DOI: $10.3892 / b r .2022 .1517$

\begin{abstract}
Since late December 2019, severe acute respiratory syndrome coronavirus 2 has spread across the world, which resulted in the World Health Organization declaring a global pandemic. Coronavirus disease 2019 (COVID-19) presents a
\end{abstract}

Correspondence to: Dr Federica Cosentino, Unit of Infectious Diseases, Department of Clinical and Experimental Medicine, ARNAS Garibaldi Nesima Hospital, University of Catania, Via Palermo 636, I-95122 Catania, Italy

E-mail: federicacosentino91@gmail.com

Abbreviations: ACE2, angiotensin-converting enzyme 2; AO, Azienda Ospedaliera; AOU, AO Universitaria; ARDS, acute respiratory distress syndrome; $\mathrm{COPD}$, chronic obstructive pulmonary disease; COVID-19, coronavirus disease 2019; CPAP, continuous positive airway pressure; CRP, C-reactive protein; HFNC, high flow nasal cannula; ICU, intensive care unit; IQR, interquartile range; $\mathrm{LDH}$, lactate dehydrogenase; MOFS, multiple organ failure syndrome; NC, nasal cannula; SARS-CoV-2, severe acute respiratory syndrome coronavirus 2 ; TNF- $\alpha$, tumor necrosis factor $\alpha$; VM, venturi mask; WHO, World Health Organization

Key words: SARS-CoV-2, pandemic, epidemiological features, predictors of death highly variable spectrum with regard to the severity of illness. Most infected individuals exhibit a mild to moderate illness $(81 \%)$; however, $14 \%$ have a serious disease and $5 \%$ develop severe acute respiratory distress syndrome (ARDS), requiring intensive care support. The mortality rate of COVID-19 continues to rise across the world. Data regarding predictors of mortality in patients with COVID 19 are still scarce but are being actively investigated. The present multicenter retrospective observational study provides a complete description of the demographic and clinical characteristics, comorbidities and laboratory abnormalities in a population of 421 hospitalized patients recruited across eight infectious disease units in Southern Italy (Sicily) with the aim of identifying the baseline characteristics predisposing COVID-19 patients to critical illness or death. In this study, older age, pre-existing comorbidities and certain changes in laboratory markers (such as neutrophilia, lymphocytopenia and increased C-reactive protein levels) at the time of admission were associated with a higher risk of mortality. Male sex, on the other hand, was not significantly associated with increased risk of mortality. Symptoms such as fatigue, older age, a number of co-pathologies and use of continuous positive airway pressure were the most significant contributors in the estimation of clinical prognosis. Further research is required to better characterize the epidemiological features of COVID-19, to understand the related predictors of death and to develop new effective therapeutic strategies. 


\section{Introduction}

In late December 2019, a cluster of patients with pneumonia of unknown origin that was epidemiologically linked to a seafood and wet animal wholesale market in Wuhan, Hubei Province, was reported in China (1). On January 7, 2020, the causative pathogen was identified as a novel $\beta$-coronavirus, subsequently named severe acute respiratory syndrome coronavirus 2 (SARS-CoV-2). In the following weeks the infection spread from a single city in China to other Chinese cities and other countries around the world. On March 11, 2020, the World Health Organization declared SARS-CoV-2 outbreak a global pandemic (2).

Up until 28th of January, 2022, >370 million cases of SARS-CoV-2 infections have been reported and almost 5.7 million individuals have died worldwide. So far, a total of 11,542,793 million cases have been reported in Italy, 148,542 of which have died (3).

COVID-19 presents a highly variable spectrum of severity of illness, ranging from mild flu-like symptoms to a severe hypoxic pneumonia, which may require mechanical ventilation. Life-threatening complications may include acute respiratory distress syndrome (ARDS), multisystem organ failure, and ultimately, death (4). Most infected individuals who develop a symptomatic disease following SARS-CoV-2 infection exhibit a mild to moderate illness (81\%); however, $14 \%$ will be considered to have a serious infection and $5 \%$ progress to severe ARDS, requiring intensive care support (5).

The most common clinical manifestations of COVID-19 include fever, dry cough, headache, fatigue, dyspnea and diarrhea. Patients can also present non-typical clinical manifestations such as isolated gastrointestinal, olfactory and/or gustatory dysfunctions (6). Most patients exhibit lymphopenia on admission (7). Prothrombin time, D-dimer levels and hypersensitive troponin I are also higher in intensive care unit (ICU) patients (8). Multiple organ dysfunction may occur in COVID-19; complications include impairment of the heart, brain, lung, liver and kidney function and alteration of the coagulation system (9). Venous and arterial thromboembolic events are present in hospitalized patients with COVID-19 with a variable incidence of between $10-25 \%$ (10).

The mortality rate of COVID-19 continues to increase across the world. Data regarding predictors of mortality in patients with COVID-19 are still relatively scarce. Several comorbidities have been shown to be associated with poor outcomes in patients with COVID-19, such as hypertension, coronary heart disease and diabetes. Some laboratory parameters were also examined as predictors of death; high levels of cardiac troponin, IL-6, C-reactive protein (CRP) and D-dimer seem to be correlated with increased mortality. Older age is also associated with a higher risk of death (11).

Characterization of the epidemiological features of COVID-19 is crucial for the development and implementation of effective therapeutic strategies. Here, the results of a retrospective descriptive study of patients hospitalized in eight clinical centers in Sicily (Italy), between 1st March and 18th May 2020 are presented.

\section{Patients and methods}

Study design and data sources. The present study was a multicenter retrospective observational study. This multicenter study was coordinated by the Infectious Diseases Unit of Presidio Ospedaliero Garibaldi Nesima (Catania, Italy) and involved the Infectious Disease Units of other seven Sicilian hospitals: Azienda Ospedaliera (AO) Cannizzaro (Catania, Italy), AO Universitaria (AOU, Italy) Policlinico G. Martino (Messina, Italy), AO Gravina (Caltagirone, Italy), AO Umberto I (Enna, Italy), AOU Policlinico P. Giaccone (Palermo, Italy), AO Umberto I (Siracusa, Italy), AO Maggiore (Modica, Italy).

Clinical data were obtained from electronic medical records or charts, and were collected using a centrally designed electronic worksheet. Collected data included patients' demographics, laboratory test results, historical and current medication lists, historical and current diagnoses and clinical notes. Specifically, the following information for each patient was obtained: Hospital, date of admission and date of discharge or death, age, sex, the first recorded inpatient laboratory tests at admission [(including white blood cells, D-dimer, creatinine, CRP, ferritin, aspartate aminotransferase (AST), alanine aminotransferase (ALT), IL-6, lactate dehydrogenase (LDH)]; past and current diagnoses (heart disease, hypertension, diabetes, respiratory disease, obesity, chronic kidney disease, others); type of oxygen therapy started upon hospital admission [nasal cannula (NC), Venturi mask (VM), continuous positive airway pressure device (CPAP) or high-flow nasal cannula (HFNC) oxygenation] and current drug therapies for COVID-19 (hydroxychloroquine, azithromycin, lopinavir/ritonavir or darunavir/cobicistat, tocilizumab, corticosteroids and antibiotics). IL-6 levels were only tested in a limited number of the patients (132 patients of 421).

Acute respiratory distress syndrome has been defined using the Berlin Definition, as lung injury of acute onset, bilateral opacities on chest imaging (chest radiograph or computed tomography) not explained by another lung pathology, respiratory failure not explained by heart failure or volume overload, decreased $\mathrm{PaO}_{2} / \mathrm{FiO}_{2}$ ratio $\left(\mathrm{PaO}_{2} / \mathrm{FiO}_{2}<300 \mathrm{mmHg}\right)$ (12).

Study cohort. Hospitalized patients were included in this study if they fulfilled two primary criteria: Age $>18$ years and having a confirmed diagnosis of SARS-CoV-2 infection by a PCR test of a nasopharyngeal sample. A total of 421 patients were recruited. The median age of the included patients was 66 years (IQR 55-78 years), ranging from 18-100 years; $235(55.8 \%)$ were male and 186 were female (44.2\%).

Ethical approval. This research was conducted according to the Declaration of Helsinki (13). It was approved as a retrospective minimally invasive experimental study by the Provincial Review Board of Catania on August 4th, 2020 (approval no. 492). Patients provided signed written informed consent for the use of their data and samples for research purposes at admission. All patients were $>18$ years of age. Each Infectious Diseases Unit provided data from hospitalized patients who had a positive test result for the SARS-CoV-2 virus between 1st March and 18th May 2020. The data were collected by a member of the medical team who treated the patient and all data were codified and anonymized before statistical analysis.

Outcomes. The registered epidemiological data, demographics, signs and symptoms on admission (fever, chills, cough, dyspnea, ageusia, anosmia), pre-existing comorbidities, 
baseline laboratory tests results, chest radiographic findings at baseline and during follow-up, treatment received for COVID-19, highest level of respiratory support, and clinical outcomes were recorded. The primary endpoint was to correlate the clinical outcome of hospitalized patients with SARS-CoV-2 infection, in terms of recovery and death, to the clinical-laboratory characteristics of the disease and the pre-existing comorbidities.

The secondary endpoint was to identify the predictors of poor outcomes using a machine learning model opening up the possibility for patient stratification and treatment allocation.

Data analysis. Continuous variables are presented as the median and interquartile range (IQR). Categorical variables are expressed as the number of patients and percentage. The baseline demographic and clinical-epidemiological characteristics of patients who recovered and those with worse outcomes (ICU or death) were compared. $\mathrm{P}<0.05$ was considered to indicate a statistically significant difference.

A Rényi test was used to compare survival. To identify potential non-linear relations and interaction effects among the investigated variables with the risk of death, machine learning analysis was used to measure the predictive power of a potential classification algorithm for the risk of death and to establish variable importance in more complex mortality prediction models. Risk models have been developed using decision-tree induction from class-labelled training records and Cox proportional hazard models. The training set was composed of records in which one attribute (such as the presence or absence of carotid atherosclerosis) was the dependent variable, and the remaining attributes were the predictor variables; the individual records are the tuples for which the class label is known. To establish the robustness of predictor variables a K-Fold Cross Validation was performed. The prediction quality of Decision Tree analysis has performed using Receiver Operating Characteristic (ROC) curve analysis. Statistical analysis was performed with the use of tools for survival analysis and recursive partitioning analysis within the R Statistical Software (14).

\section{Results}

Between March 1st and May 18th 2020, a total of 421 patients diagnosed with COVID-19 and hospitalized at one of eight clinical centers across Sicily were included in this retrospective analysis. Table I shows the baseline characteristics of the patients. Distribution of infected people and the median age of each group is shown in Fig. 1.

On admission, most patients had fever $(n=334,79.3 \%)$, dyspnea $(n=195,46.3 \%)$ and fatigue $(n=120,28.5 \%)$. The other symptoms included cough $(\mathrm{n}=195,46.3 \%)$, ageusia $(n=10,2.4 \%)$, and anosmia $(n=10,2.4 \%) ; 47$ of $421(11.2 \%)$ patients were asymptomatic from exposure to admission.

Baseline laboratory findings are shown in Table II. The most common abnormalities in blood counts included lymphopenia (61.1\%) and neutrophilia (27.1\%). Additionally, $55.7 \%$ had D-dimer levels above the normal range. High serum levels were reported for ALT (12.2\%), AST (38.8\%) and CRP $(83.2 \%)$. Ferritin and IL-6 were tested in a limited number of patients, and high concentrations of these
Table I. Baseline characteristics of patients hospitalized with COVID-19.

\begin{tabular}{|c|c|}
\hline Parameter & No. $(\%)$ \\
\hline Total N & 421 \\
\hline Age, years, median (range) & $66(18-100)$ \\
\hline \multicolumn{2}{|l|}{ Sex } \\
\hline Female & $186(44.2 \%)$ \\
\hline Male & $235(55.8 \%)$ \\
\hline Asymptomatic & $47(11.2 \%)$ \\
\hline Symptoms & $373(88.6 \%)$ \\
\hline Fever & $334(79.3 \%)$ \\
\hline Dyspnoea & $195(46.3 \%)$ \\
\hline Asthenia & $120(28.5 \%)$ \\
\hline Cough & $195(46.3 \%)$ \\
\hline Ageusia & $10(2.4 \%)$ \\
\hline Anosmia & $10(2.4 \%)$ \\
\hline Comorbidity & $345(82 \%)$ \\
\hline Hypertension & $227(53.9 \%)$ \\
\hline Cardiovascular disease & $115(27.3 \%)$ \\
\hline Diabetes & $88(20.9 \%)$ \\
\hline $\mathrm{BPCO}$ & $50(11.9 \%)$ \\
\hline Chronic kideney disease & $36(8.6 \%)$ \\
\hline Obesity & $69(16.4 \%)$ \\
\hline Others (cancer, rheumatic disorders, etc.) & $242(57.4 \%)$ \\
\hline \multicolumn{2}{|l|}{ Oxygen Requirement } \\
\hline Continuous positive airway pressure & $69(16.3 \%)$ \\
\hline High flow nasal cannula & $36(8.5 \%)$ \\
\hline Venturi mask or nasal cannula & $284(67.4 \%)$ \\
\hline
\end{tabular}

parameters were found in 100 of $137(73 \%)$ and 79 of 136 $(58 \%)$, respectively.

A total of $312(74 \%)$ patients had findings of bilateral infiltrates on radiographic imaging, while $28(6.6 \%)$ patients had unilateral infiltrates; $42(10 \%)$ patients had no evidence of pneumonia on admission. Of the 421 patients, $289(68.6 \%)$ required oxygen support in the hospital; of these, $69(16.3 \%)$ received oxygen therapy with CPAP, 36 (8.5\%) with HFNC, and $284(67.4 \%)$ with VM or NC. During hospitalization, $317(75 \%)$ of the 421 patients developed ARDS, and 21 of these patients with ARDS were admitted to ICUs; 5 patients died in the Infectious Disease Units. Overall mortality increased with age $(88.6 \%$ of deaths occurred in people $>75$ years). Of the total cohort, $346(82.2 \%)$ patients achieved clinical recovery, $31(7.4 \%)$ patients were transferred to an ICU and $44(10.4 \%)$ died during hospitalization in the Infectious Disease Unit.

Of the 421 patients recruited, $252(59.8 \%)$ received treatment with hydroxychloroquine plus azithromycin, 88 (20.9\%) patients received hydroxychloroquine alone, 120 (28.5\%) patients received lopinavir/ritonavir, 78 (18.5\%) patients received darunavir/cobicistat and $86(20.4 \%)$ patients received tocilizumab; 294 (70\%) also underwent antibiotic therapy.

Overall survival did not show statistically significant differences between the two sexes $(\mathrm{P}=0.9)$. Overall survival 


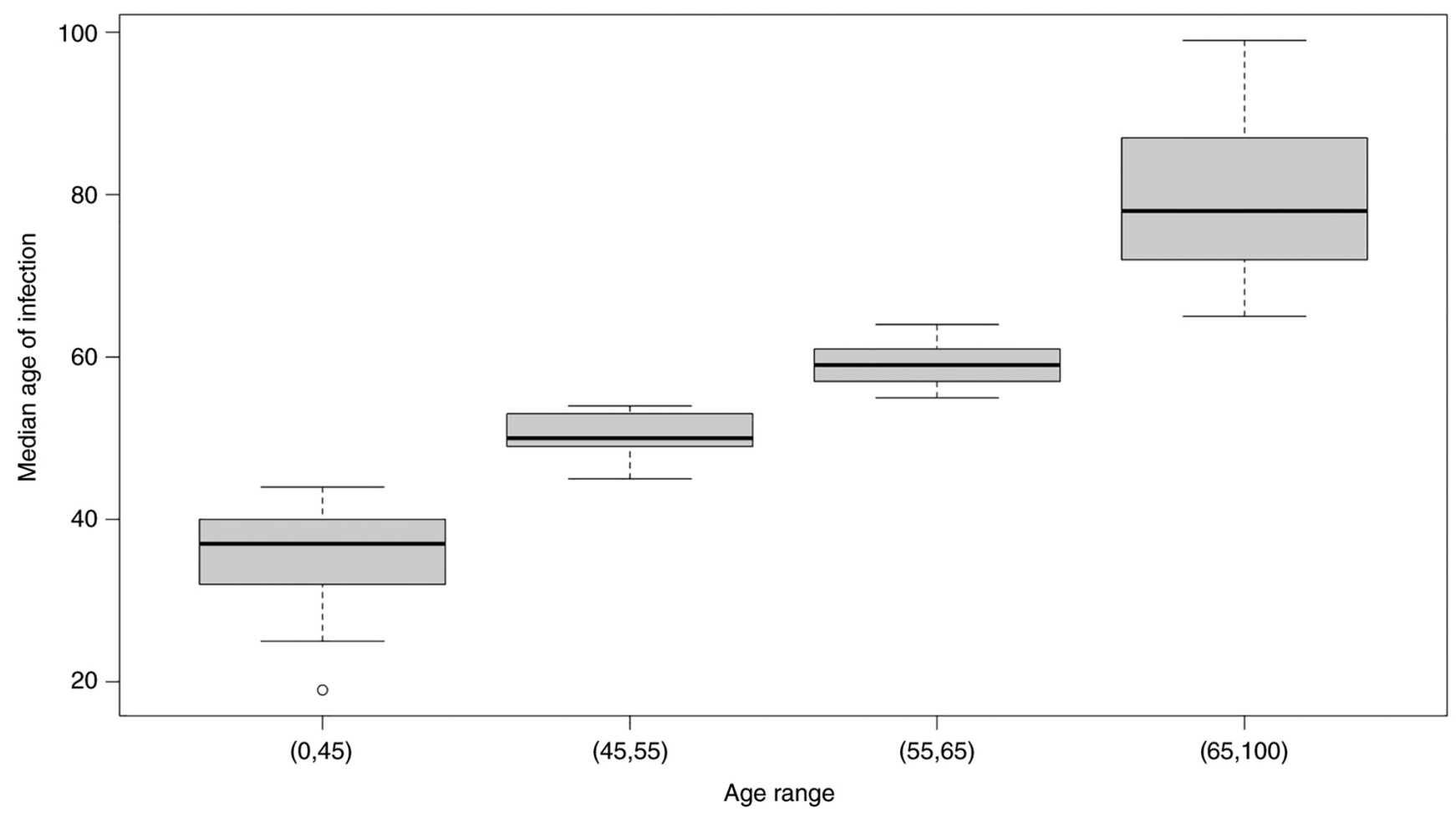

Figure 1. Distribution of infected people and median age of each group.

in relation to age groups, on the other hand, highlighted how age represents one of the main predictors of an unfavorable outcome, with an increased risk for people aged $>75$ years (P<0.0001, Fig. 2).

The majority of the patients $(n=345,82 \%)$ had at least one comorbidity, including hypertension $(\mathrm{n}=227,53.9 \%)$, cardiovascular diseases $(n=115,27.3 \%)$, diabetes $(n=88,20.9 \%)$, chronic obstructive pulmonary disease (COPD) $(n=50,11.9 \%)$, chronic kidney disease $(n=36,8.6 \%)$, obesity $(n=69,16.4 \%)$, and others (including cancer, rheumatic disorders, etc.) $(n=242,57.4 \%)$. The presence of comorbidities was associated with a reduced survival in our population. Specifically, the greatest significance was found for hypertension $(\mathrm{P}=0.000091)$, heart disease $(\mathrm{P}=0.0008)$ and chronic renal failure $(\mathrm{P}=0.002)$, while the data analysis showed how the presence of diabetes is only slightly statistically significant for worse outcome. $(\mathrm{P}=0.031)$.

A first risk model was developed to identify those variables that in the sample mostly influenced the outcome of the patient. The patients were stratified into two groups: The first group included those who recovered, and the second group consisted of patients with a negative outcome (ICU or death). A model including all the hematological and clinical variables associated with each patient was developed. To understand the effect of comorbidities, a variable storing the number of comorbidities each patient had was defined. Age was stratified as follows: $<45$ years, $45-55,56-65$ and $>66$. The variables that significantly were able to predict the outcome were: Fatigue, CPAP, age and number of pathologies. The generated model had an area under the ROC curve of 0.82 , a sensitivity of 0.42 and a specificity equal to 0.97 . Although the sensitivity of the model is low, the results show that predicting variables have a clear role in the outcome of the illness. The results are presented in Fig. 3.

\section{Discussion}

More than a year after its onset, the SARS-CoV-2 pandemic still represents a global emergency, and it accounts for serious clinical complications in 10-20\% of infected subjects. Since the beginning of the pandemic, Italy has been heavily affected.

Data from Northern Italy have found that patients with concomitant cardiac disease and COVID-19 have a poor prognosis compared with subjects without a history of cardiac disease (15). A study that included 1,761 patients from 13 regions in Italy showed that age and comorbidities are the most important predictors of death among COVID-19 patients (16).

The present multicenter retrospective observational study provides a complete description of the demographic and clinical characteristics, comorbidities and laboratory abnormalities in a cohort of 421 hospitalized patients recruited in eight Infectious Disease Units in Southern Italy (Sicily) and aims to identify the baseline characteristics predisposing COVID-19 patients to critical illness or death.

The median age of the included patients was 66 years (IQR 55-78 years), which was similar to that reported in other studies (17,18). Similar to data from China (19), those collected in the present study relating to demographic factors showed how old age is significantly associated with a reduced probability of survival. This evidence supports research findings that outline how age is one of the most important predictors of serious illness and mortality. It is likely that age-related alterations in immunological functions and type 2 cytokine production seem to lead to deficiencies in controlling SARS-CoV-2 replication and pro-inflammatory responses, as suggested by Zhou et al (20). 
Table II. Laboratory results of patients hospitalized with COVID-19.

\begin{tabular}{lccc}
\hline Blood parameter & Mean & No. $(\%)$ & Reference ranges \\
\hline White blood cell count, cells $/ \mathrm{mm}^{3}$ & $7,126.75$ & $137(32.5 \%)$ & $4,300-10,300$ \\
Neutrophils, cells $/ \mathrm{mm}^{3}$ & $5,143.34$ & $95(27.1 \%)$ & $2,100-6,100$ \\
Lymphocytes, cells $/ \mathrm{mm}^{3}$ & $1,216.55$ & $248(61.1 \%)$ & $1,300-3,500$ \\
C-reactive protein, $\mathrm{mg} / \mathrm{dl}$ & 6.95 & $346(83.2 \%)$ & $0.01-0.50$ \\
Ferritin, ng/ml & 758.93 & $100(73 \%)$ & $21-275$ \\
Lactate dehydrogenase, UI/l & 455.27 & $215(81.4 \%)$ & $125-243$ \\
Aspartate aminotransferase, UI/l & 38.79 & $163(38.8 \%)$ & $5-34$ \\
Alanine aminotransferase, UI/l & 37.50 & $51(12.2 \%)$ & $0-55$ \\
D-dimer, ng/ml & $1,582.12$ & $200(55.7 \%)$ & $0-500$ \\
IL-6, pg/ml & 115.15 & $79(58.0 \%)$ & $0-19$ \\
\hline
\end{tabular}

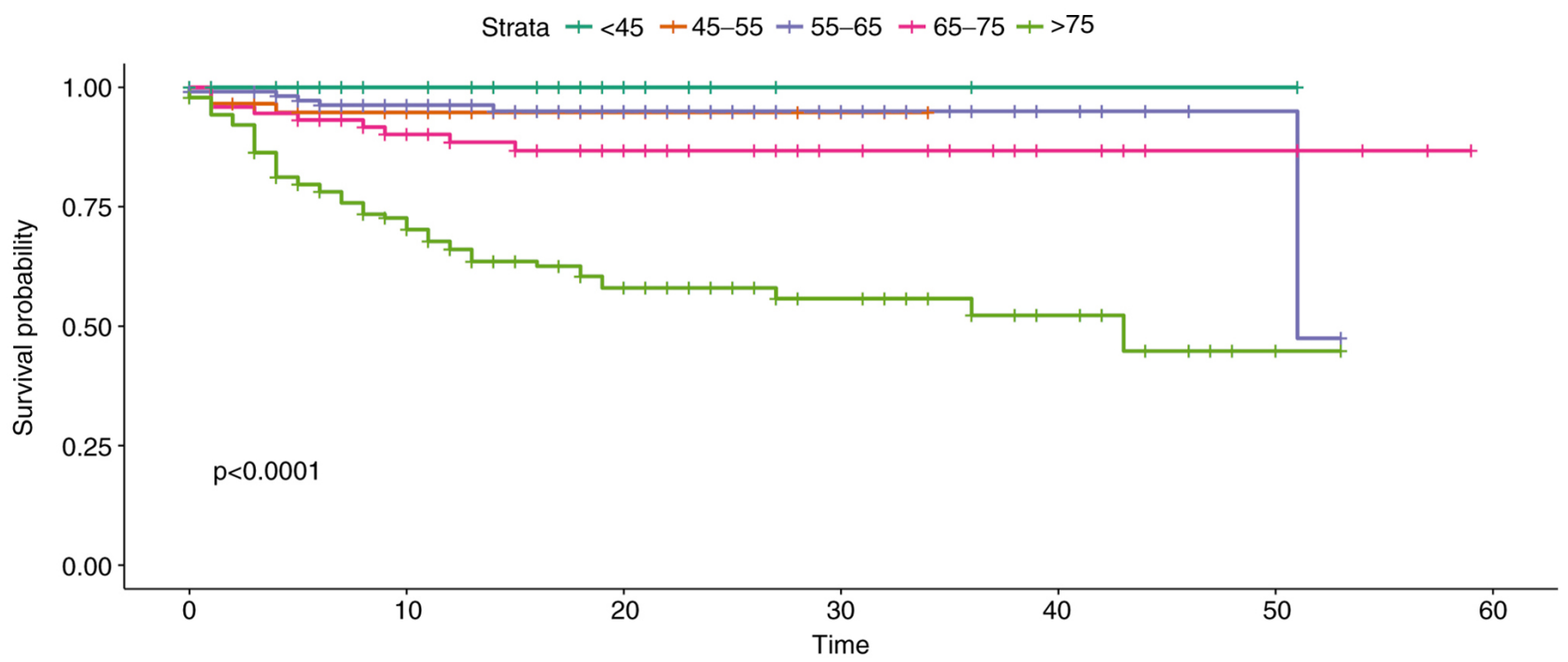

\begin{tabular}{|c|c|c|c|c|c|c|c|}
\hline \multicolumn{8}{|c|}{ Number at risk } \\
\hline$<45-$ & 38 & 23 & 7 & 2 & 1 & 1 & 0 \\
\hline ๘ 45-55- & 58 & 43 & 26 & 6 & 0 & 0 & 0 \\
\hline స్ㅠ 55-65- & 110 & 86 & 48 & 23 & 7 & 2 & 0 \\
\hline ๘ 65-75- & 73 & 58 & 39 & 17 & 9 & 5 & 0 \\
\hline$>75$ & 140 & 90 & 48 & 22 & 9 & 2 & 0 \\
\hline & 0 & 10 & 20 & $\begin{array}{l}30 \\
\text { Time }\end{array}$ & 40 & 50 & 60 \\
\hline
\end{tabular}

Figure 2. Survival probability in relation to age groups. Patients have been stratified and compared according to age in the following groups: $<45$ years, $45-55$ years, $56-65$ years, $66-75$ years, $>75$ years.

In contrast to other studies, which have suggested that male sex may be associated with a higher risk of developing severe and fatal COVID-19, the incidence of illness was similar in the two sexes in the present study with no significant differences in terms of overall survival (21).

To date, several studies have reported the important relationships between COVID-19 severity and mortality and specific pathologies, partly supporting the present research. A meta-analysis based on data from 7 studies in China found that hypertension, diabetes, respiratory system disease and cardiovascular disease were more frequent in severe patients (22). Another systematic review of 14 articles involving 4,659 patients, observed that hypertension increases the risk of death from COVID-19 by $>2.5$-fold (11). The mechanisms underlying the association between cardiovascular disease and COVID-19 remain unclear, but it may be due to infection-related ischemia that progresses to myocardial injury and/or a viral-induced inflammatory storm causing shock and the ensuing ischemic-related injury. In addition, a direct myocardial invasion of the virus mediated by ACE2 receptors may be involved, and a hypercoagulable state with thrombotic disorders were observed in certain patients. A possible association between SARS-CoV-2 infection and cardiovascular events has been recently hypothesized in a case report of a 


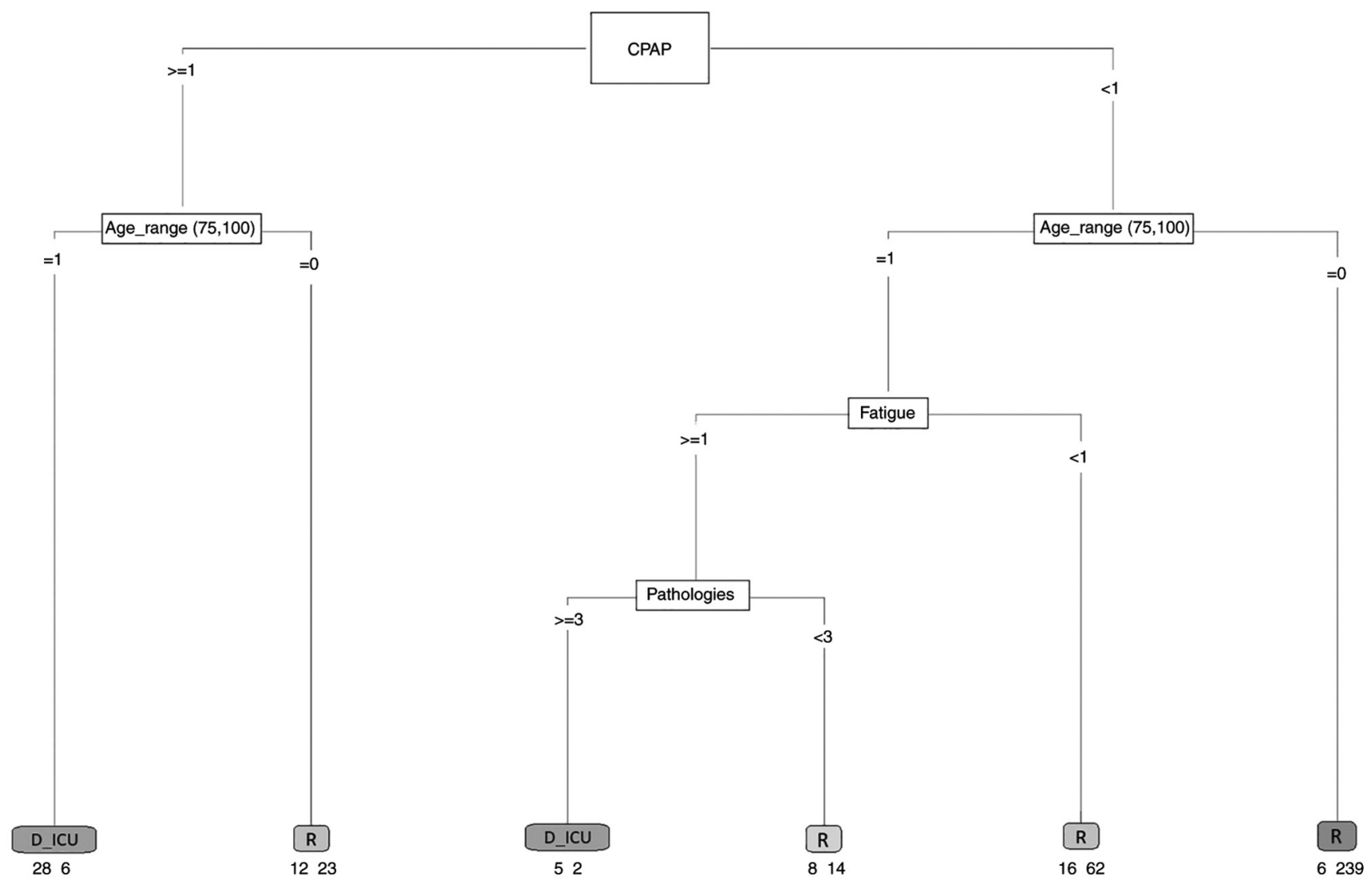

Figure 3. Decision tree algorithm. The decision tree contains a root node, internal nodes and leaf or terminal nodes. Each leaf is assigned for a class label. The numbers under the leaves represent the output result. For example, D_ICU 28-6 indicates that there were 34 subjects in this class: 28 individuals who died or were transferred to the ICU and 6 individuals who recovered. CPAP, continuous positive airway pressure; R, recovered; D_ICU, died or were transferred to the intensive care unit.

59-year-old woman affected by hypertension and metabolic disorders, treated for a SARS-CoV-2 infection, who developed cardiac symptoms during the first days of hospitalization (23).

Diabetes was not significantly associated with severe COVID-19 infection-associated illness in the present study, contrary to other meta-analyses (24) that have found how people affected have a greater risk of severe/critical illness and in-hospital mortality in diabetics. A possible explanation of this finding is that there was a large percentage of individuals with undiagnosed diabetes at admission in our hospitals. Several factors seem to increase the risk of severe COVID-19 in diabetic subjects: Diabetics often have compromised innate immunity, and thus, they are more prone to contracting infections; furthermore, exaggerated expression of pro-inflammatory cytokines in these subjects may contribute to the cytokine storm that is observed in severe cases of COVID-19 (25).

Finally, it has been reported that patients with chronic kidney disease exhibit increased expression of ACE2 (26), which may promote an increase in viral load; moreover, considering the possibility of harmful effects of the virus on the renal system, patients with pre-existing chronic kidney disease have a higher risk of mortality.

The majority of the patients in the present cohort had abnormal laboratory tests at baseline. The analysis of the data showed that patients with sustained systemic inflammatory response had more unfavorable outcomes. Elevated values of CRP were associated with a higher risk of death or transfer to an
ICU in the present study. Increased values of LDH and ferritin were not significantly associated with a poorer outcome in the present study. In line with these findings, Luo et al (27) found that serum CRP levels upon admission were independently associated with adverse outcomes of COVID-19. It has been shown that in pulmonary diseases marked by inflammatory features, for example there is a typical increase in serum CRP levels in response to inflammatory cytokines (27). Indeed, in individuals affected by SARS-CoV-2, there is a dysregulated production of cytokines such as IL-6, TNF- $\alpha$ and chemokines that can lead to ARDS, MOFS and recall of inflammatory cells in the lungs with development of oedema and alveolar damage (28). Elevated values of IL-6 were associated with a higher risk of death and transfer to ICU in the present cohort, although IL-6 levels were only tested in a limited number of the patients (132 patients of 421).

Patients with lymphopenia and abnormal values of neutrophils at the time of admission, had a worse outcome in the present study. The baseline neutrophil-to-lymphocyte ratio has been confirmed as a potential predictor for critical illness of COVID-19 (29). The exact relationship behind this correlation remains unclear.

To take into account potential non-linear relations and interaction effects among clinical and demographic risk factors for severity of COVID-19, an attempt to estimate mortality risk in COVID-19 patients using a machine learning algorithm was performed. Although the model exploited a limited sample size and the sensitivity was low, the results 
clearly showed that predictive variables such as age, certain symptoms like fatigue, other pre-existing pathologies and use of CPAP have a clear role in the outcome of the illness.

At the beginning of the pandemic, international guidelines did not recommend the use of CPAP in COVID-19 patients because of the lack of randomized studies showing its efficacy, concerns about infection dissemination and a possible delayed endotracheal intubation. Therefore, CPAP was the object of conflicting evidence. To date, its value in COVID-19 treatment remains to be established. Early after the COVID-19 outbreak in Italy, there was an expert consensus in favor of CPAP and non-invasive ventilation as first-line treatments for the associated acute hypoxemic respiratory failure (30). Ashish et al (31) found that use of CPAP early in the course of a patient's admission is associated with reduced mortality, whereas use of CPAP in patients with a longer stay in hospital was associated with increased mortality (31).

Compared with CPAP therapy, the use of HFNC has been associated in some studies with a lower mortality rate in hypoxemic respiratory failure and with a decreased need of subsequent intubation and ICU admission (32). Furthermore, HFNC has been shown to be associated with a significantly lower risk of bioaerosol dispersion, reducing the risk of hospital-acquired infections for health workers (33). Case reports and case series have demonstrated how patients may achieve a marked improvement in respiratory function, as well as lower respiratory fatigue, with better results on arterial gas analysis when treated early with $\operatorname{HFNC}(34,35)$.

Further studies are needed to clarify if CPAP or High-Flow Nasal Oxygen are clinically effective when compared with standard oxygen therapy in patients with confirmed COVID-19.

The present study has some limitations that should be addressed. Firstly, this was an observational, retrospective analysis. Furthermore, although it was a multicenter study, it consists of a limited sample size and patients belong to a small geographic area that could not entirely reflect the international situation. Finally, certain data regarding the clinical and laboratory status of patients were missing in the medical records.

COVID-19 has become a worldwide pandemic affecting $>370$ million individuals. In 10-20\% of COVID-19 patients, their condition deteriorates to severe or critical illness, characterized by ARDS and multiorgan dysfunction syndrome. No effective treatments for severe patients have yet been identified. Early identification of patients at risk of adverse outcomes has the potential to enable more individualized treatment strategies and to more efficiently utilize health care resources, increasing the number of lives saved. In the present study, older age, pre-existing comorbidities (hypertension, heart disease, chronic renal failure and COPD) and some changes in laboratory markers at the time of admission (neutrophilia, lymphocytopenia, increased CRP), were associated with a higher risk of mortality. Male sex, on the other hand, did not reach statistical significance.

The extensive actions taken by governments have slowed down the epidemic; and vaccines and monoclonal antibodies have further curbed the spread of COVID-19 worldwide. Although the epidemic appears to be in decline in almost all regions of the world, we may yet face further challenges. Understanding which factors increase the risk of poor outcome will help us to better face these challenges.
In conclusion, several prognostic models in predicting adverse outcomes have been proposed, and the aim of the present study was to identify the patients who were likely to develop them. A machine learning model incorporating demographic, clinical and laboratory variables was established. Symptoms such as fatigue, older age, number of co-pathologies and use of CPAP were the most significant contributors in the estimation of clinical prognosis.

\section{Acknowledgements}

We would like to thank Dr Pietro Leanza (University of Catania, Catania, Italy) for his assistance revising the manuscript.

\section{Funding}

No funding was received.

\section{Availability of data and materials}

The datasets used and/or analyzed during the present study are available from the corresponding author on reasonable request.

\section{Authors' contributions}

FC wrote the paper. FC, VM, AM, APa, DS, RFDV and DL collected the data. FB, MG, LL, VB, GV, AZ, AO, CI, SB, $\mathrm{LG}, \mathrm{AC}, \mathrm{AF}$ and MADR provided clinical assistance to the patients. APu performed the data analysis. MC, RB, SG, ST and GL searched literature references. GN, BC and BMC critically revised the manuscript. All authors have read and approved the final manuscript. BC and BMC confirm the authenticity of all the raw data.

\section{Ethics approval and consent to participate}

This research was conducted according to the Declaration of Helsinki. It was approved as a retrospective minimally invasive experimental study by the Provincial Review Board of Catania on August 4th, 2020 (approval no. 492). Patients provided signed written informed consent for the use of their data and samples for research purposes at admission.

\section{Consent for publication}

Not applicable.

\section{Competing interests}

The authors declare that they have no competing interests.

\section{References}

1. Zhu N, Zhang D, Wang W, Li X, Yang B, Song J, Zhao X, Huang B, Shi W, Lu R, et al: A novel coronavirus from patients with Pneumonia in China, 2019. N Engl J Med 382: 727-733, 2020.

2. World Health Organization (WHO): Rolling updates on coronavirus disease (COVID-19). In: Events as they happen. World Health Organization, Geneva, Switzerland, 2020.

3. World Health Organization (WHO): Novel Coronavirus (COVID-19). In: Situation reports. World Health Organization, Geneva, Switzerland, 2020. 
4. Epidemiology Working Group for NCIP Epidemic Response, Chinese Center for Disease Control and Prevention: The epidemiological characteristics of an outbreak of 2019 novel coronavirus diseases (COVID-19) in China. Zhonghua Liu Xing Bing Xue Za Zhi 41: 145-151, 2020 (In Chinese).

5. Wu Z and McGoogan JM: Characteristics of and important lessons from the coronavirus disease 2019 (COVID-19) outbreak in China: Summary of a Report of 72314 cases from the Chinese center for disease control and prevention. JAMA 323: 1239-1242, 2020.

6. Cevik M, Kuppalli K, Kindrachuk J and Peiris M: Virology, transmission, and pathogenesis of SARS-CoV-2. BMJ 371: m3862, 2020 .

7. Henry B, Cheruiyot I, Vikse J, Mutua V, Kipkorir V, Benoit J, Plebani M, Bragazzi N and Lippi G: Lymphopenia and neutrophilia at admission predicts severity and mortality in patients with COVID-19: A meta-analysis. Acta Biomed 91: e2020008, 2020

8. Wang D, Hu B, Hu C, Zhu F, Liu X, Zhang J, Wang B, Xiang H, Cheng Z, Xiong Y, et al: Clinical characteristics of 138 hospitalized patients with 2019 novel coronavirus-infected Pneumonia in Wuhan, China. JAMA 323: 1061-1069, 2020.

9. Zaim S, Chong JH, Sankaranarayanan V and Harky A: COVID-19 and multiorgan response. Curr Probl Cardiol 45: 100618, 2020.

10. Wiersinga WJ, Rhodes A, Cheng AC, Peacock SJ and Prescott HC: Pathophysiology, transmission, diagnosis, and treatment of coronavirus disease 2019 (COVID-19): A review. JAMA 324: 782-793, 2020

11. Tian W, Jiang W, Yao J, Nicholson CJ, Li RH, Sigurslid HH Wooster L, Rotter JI, Guo X and Malhotra R: Predictors of mortality in hospitalized COVID-19 patients: A systematic review and meta-analysis. J Med Virol 92: 1875-1883, 2020.

12. ARDS Definition Task Force, Ranieri VM, Rubenfeld GD Thompson BT, Ferguson ND, Caldwell E, Fan E, Camporota L and Slutsky AS: Acute respiratory distress syndrome: The Berlin definition. JAMA 307: 2526-2533, 2012.

13. World Medical Association: World Medical Association Declaration of Helsinki: Ethical principles for medical research involving human subjects. JAMA 310: 2191-2194, 2013.

14. RStudio Team: Integrated development for R. RStudio, Inc., Boston, MA, 2015. http://www.rstudio.com/.

15. Inciardi RM, Adamo M, Lupi L, Cani DS, Di Pasquale M, Tomasoni D, Italia L, Zaccone G, Tedino C, Fabbricatore D, et al: Characteristics and outcomes of patients hospitalized for COVID-19 and cardiac disease in Northern Italy. Eur Heart J 41: $1821-1829,2020$

16. Iaccarino G, Grassi G, Borghi C, Ferri C, Salvetti M and Volpe M; SARS-RAS Investigators: Age and Multimorbidity Predict Death Among COVID-19 Patients: Results of the SARS-RAS Study of the Italian Society of Hypertension. Hypertension 76: 366-372, 2020

17. Richardson S, Hirsch JS, Narasimhan M, Crawford JM, McGinn T, Davidson KW; The Northwell COVID-19 Research Consortium, Barnaby DP, Becker LB, Chelico JD, et al: Presenting characteristics, comorbidities, and outcomes among 5700 patients hospitalized with COVID-19 in the New York City Area. JAMA 323: 2052-2059, 2020

18. Giacomelli A, Ridolfo AL, Milazzo L, Oreni L, Bernacchia D, Siano M, Bonazzetti C, Covizzi A, Schiuma M, Passerini M, et al: 30-day mortality in patients hospitalized with COVID-19 during the first wave of the Italian epidemic: A prospective cohort study. Pharmacol Res 158: 104931,2020.

19. Wu C, Chen X, Cai Y, Xia J, Zhou X, Xu S, Huang H, Zhang L, Zhou X, Du C, et al: Risk factors associated with acute respiratory distress syndrome and death in patients with coronavirus disease 2019 Pneumonia in Wuhan, China. JAMA Intern Med 180: 934-943, 2020.

20. Zhou F, Yu T, Du R, Fan G, Liu Y, Liu Z, Xiang J, Wang Y, Song B, Gu X, et al: Clinical course and risk factors for mortality of adult inpatients with COVID-19 in Wuhan, China: A retrospective cohort study. Lancet 395: 1054-1062, 2020.
21. Wenham C, Smith J and Morgan R; Gender and COVID-19 Working Group: COVID-19: The gendered impacts of the outbreak. Lancet 395: 846-848, 2020.

22. Yang J, Zheng Y, Gou X, Pu K, Chen Z, Guo Q, Ji R, Wang H, Wang Y and Zhou Y: Prevalence of comorbidities and its effects in patients infected with SARS-CoV-2: A systematic review and meta-analysis. Int J Infect Dis 94: 91-95, 2020.

23. Ceccarelli M, Marino A, Cosentino F, Moscatt V, Celesia BM, Gussio M, Bruno R, Rullo EV, Nunnari G and Cacopardo BS Post-infectious ST elevation myocardial infarction following a COVID-19 infection: A case report. Biomed Rep 16: 10, 2022.

24. Gold MS, Sehayek D, Gabrielli S, Zhang X, McCusker C and Ben-Shoshan M: COVID-19 and comorbidities: A systematic review and meta-analysis. Postgrad Med 132: 749-755, 2020.

25. Mantovani A, Byrne CD, Zheng MH and Targher G: Diabetes as a risk factor for greater COVID-19 severity and in-hospital death: A meta-analysis of observational studies. Nutr Metab Cardiovasc Dis 30: 1236-1248, 2020.

26. Ejaz H, Alsrhani A, Zafar A, Javed H, Junaid K, Abdalla AE, Abosalif KOA, Ahmed Z and Younas S: COVID-19 and comorbidities: Deleterious impact on infected patients. J Infect Public Health 13: 1833-1839, 2020

27. Luo X, Zhou W, Yan X, Guo T, Wang B, Xia H, Ye L, Xiong J, Jiang Z, Liu Y, et al: Prognostic Value of C-Reactive Protein in Patients with Coronavirus 2019. Clin Infect Dis 71: 2174-2179, 2020.

28. Shah VK, Firmal P, Alam A, Ganguly D and Chattopadhyay S: Overview of immune response during SARS-CoV-2 infection: Lessons from the past. Front Immunol 11: 1949, 2020

29. Liu J, Liu Y, Xiang P, Pu L, Xiong H, Li C, Zhang M, Tan J, Xu Y, Song R, et al: Neutrophil-to-lymphocyte ratio predicts critical illness patients with 2019 coronavirus disease in the early stage. J Transl Med 18: 206, 2020.

30. Vitacca M, Nava S, Santus P and Harari S: Early consensus management for non-ICU acute respiratory failure SARS-CoV-2 emergency in Italy: From ward to trenches. Eur Respir J 55: 2000632, 2020.

31. Ashish A, Unsworth A, Martindale J, Sundar R, Kavuri K, Sedda L and Farrier M: CPAP management of COVID-19 respiratory failure: A first quantitative analysis from an inpatient service evaluation. BMJ Open Respir Res 7: e000692, 2020.

32. Rochwerg B, Granton D, Wang DX, Helviz Y, Einav S, Frat JP, Mekontso-Dessap A, Schreiber A, Azoulay E, Mercat A, et al: High flow nasal cannula compared with conventional oxygen therapy for acute hypoxemic respiratory failure: A systematic review and meta-analysis. Intensive Care Med 45: 563-572, 2019.

33. Li J, Fink JB and Ehrmann S: High-flow nasal cannula for COVID-19 patients: Low risk of bio-aerosol dispersion. Eur Respir J 55: 2000892, 2020.

34. Marino A, Pampaloni A, Scuderi D, Cosentino F, Moscatt V, Ceccarelli M, Gussio M, Celesia BM, Bruno R, Borraccino S, et al: High-flow nasal cannula oxygenation and tocilizumab administration in patients critically ill with COVID-19: A report of three cases and a literature review. World Acad Sci J 2: 23, 2020.

35. Marino A, Cosentino F, Pampaloni A, Scuderi D, Moscatt V, Ceccarelli M, Gussio M, Onorante A, Zagami A, Torrisi S, et al: Role of tocilizumab and high flow nasal cannula in the clinical management of severe covid-19. J Clin Trials 10: 427, 2020.

This work is licensed under a Creative Commons Attribution-NonCommercial-NoDerivatives 4.0 International (CC BY-NC-ND 4.0) License. 